\title{
Validation and analysis of the futurebus arbitration protocol: A case study
}

\author{
F BOUSSINOT $^{+}$, S RAMESH $^{\ddagger}$, R K SHYAMASUNDAR $^{\S}$ and \\ R DE SIMONEII
}

${ }^{+}$Ecole des Mines, CMA, Sophia Antipolis 06904 Sophia-Antipolis CDX, France

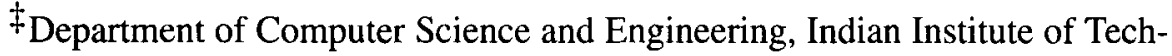
nology, Powai, Bombay 400 076, India

$\S$ Tata Institute of Fundamental Research, Bombay 400 005, India

IINRIA, 06904 Sophia Antipolis CDX, France

\begin{abstract}
In this paper, we use perfectly synchronous languages such as EsTEREL, for modelling Futurebus arbitration protocol. We show that the perfect synchrony aids in the formalization, testing, validating and verifying the protocol. We discuss solutions to the above protocol and show that properties such as mutual exclusion and deadlock-freedom can be established formally. Further, we show how the simulators can be used for testing and validation and can verify an instantiation of the protocol through algebraic tools such as auto/autograph.
\end{abstract}

Keywords. Synchronous languages; futurebus arbitration protocol; Esterel.

\section{Introduction}

The concurrency and reactive behaviour intrinsic in the futurebus protocol makes it natural to think of modelling the protocol in the following classes of languages:

1. Asynchronous languages: Here, a program is treated as a set of loosely coupled independent execution units or processes, each process evolving at its own pace. Interprocess communication is done by mechanisms such as message passing. Communication as a whole is asynchronous in the sense that an arbitrary amount of time can pass between the desire of communication and its actual completion. This class includes languages such as Ada, Occam, CSP etc.

2. Perfectly synchronous languages: In this class, programs react instantaneously to its inputs by producing the required outputs. Statements evolve in a tightly coupled inputdriven way deterministically and communication is done by instantaneous broadcast where the receiver receives a message exactly at the time it is sent. That is, a perfectly synchronous program produces its outputs from its input with no observable time delay. Languages such as Esterel (Berry \& Gonthier 1992; Berry 1992), Lustre (Halbwachs 
et al 1991), Signal (Le Guernic et al 1991), Statecharts (Harel 1987) belong to this category.

Thus, one can have two points of view on this protocol. The first one corresponds to asynchrony. Each module runs independently and all interleaving of actions are valid. Analysis is difficult and it is not clear at all that the protocol does what it is intended to do. The second point of view corresponds to the synchronous approach: there are common instants shared by all processes. This limits possible action interleavings and for example forbids one module to continuously progress although others remain blocked.

The synchronous approach is rather natural in an hardware implementation of the protocol. In this context, global instants exist and are defined by reference to the basic clock. We can expect behaviours to be reproducible which certainly simplifies the debugging process. On the other hand, the synchronous hypothesis is unrealistic in software implementations where modules can be, for example, distributed through a network. In this case, (almost) all interleavings are possible and there is no hope to remain in the purely deterministic case.

In this paper, we show how perfectly synchronous languages such as ESTEREL can be used for formal modelling, testing, validation and verification of the Futurebus protocol. We develop solutions in synchronous and asynchronous frameworks and discuss their advantages and difficulties. In particular,

1. First we describe a solution in ESTEREL wherein

- a module which is a Master Elect captures the bus and releases it immediately (so that, there is no clear distinction between the Master and the Master Elect).

- a module can enter into competition at any point.

Later, we refine the solution to have a distinction between the Master Elect and the Master. After showing the correspondence of the solution with the specification of the protocol, we show that the solution satisfies the properties of mutual exclusion and deadlock freedom. However, the solution suffers from the possibility of a livelock due to the preemption specification in the protocol.

2. Then, we describe an asynchronous solution using another reactive language RC (Boussinot 1991) in a distributed setup. Here, we discuss the need of restricting the arbitration numbers to establish the properties of mutual exclusion and deadlock-freedom. We also show how the testing and formal correctness at least for an instantiation of the protocol helps in gaining the confidence of the solution. For the latter purpose (that is for formal correctness), we use the auto/autograph (Roy \& de Simone 1990).

\section{Futurebus arbitration protocols}

Futurebus + (IEEE 1991), is a set of tools with which to implement a bus architecture providing performance scalability over both cost and time for multiple generations of single and multiple-bus multiprocessor systems. The specification of arbitration specification plays a crucial role in the performance of the system. In the following, we describe the arbitration process as in IEEE (1991). 


\subsection{Arbitration process}

1. When a module needs to send data to, or obtain data from another module, it must first gain tenure of the bus. Since two or more modules may seek to gain tenure of the bus at the same time, the arbitration process is used to restrict tenure of the bus to one module at a time. Since the arbitration process operates in parallel with, and independent of the data transfer process, an arbitration competition for control of the bus may take place concurrently with transactions on the parallel highway (main bus).

2. Each module is assigned a unique arbitration number, which is used to resolve arbitration competition. When two or more modules compete for the bus, the winner is the one whose arbitration number is the largest. Parallel contention arbitration is a process whereby modules assert their unique arbitration number on the arbitration bus and release signals according to an algorithm which after a period of time will ensure that only the winner's number remains on the arbitration bus.

3. The value of the arbitration numbers used by modules in a competition determines the sequence of arbitration process. Because of limitations in the number of bus lines used for arbitration, some numbers require two passes (or more - see the step below) of the control cycle. The module with the highest arbitration number at the end of the arbitration competition is referred to as the Master Elect. A master elect can take the bus when the module using it releases it. On taking the bus, it is referred to as the Master (the competition for the bus can begin after the master elect becomes the master).

4. There may be times when a module has urgent need of the bus after a master elect has been chosen, but before the master elect has become master. If that module has a higher arbitration number than the master elect, it may initiate a new competition to establish a new master elect. This process is referred to as preemption. Preemption allows a high priority module to acquire tenure of the bus with minimum latency (although with some sacrifice to the overall performance of the system since it forces a new arbitration competition).

In the following, we describe the abstraction of the protocol with an algorithm for solving contentions for the bus. The abstraction integrates the preemption mode described above.

\subsection{Abstraction of the protocol}

There are $N$ modules running in parallel and sharing a bus made of $P$ lines. Each module is connected to the bus by, say $P$ lines. Each module has its own arbitration binary number of length equal to the number of lines $P$ ( $a$ priori given). The problem is to design a protocol such that each module can get a mutually exclusive access to the bus. The operations that can be performed by the module and the bus are given below.

Each module can perform the following operations:

- Whenever the module wants to get an access to the bus, it places its number on the lines.

- After placing its value, it can read the value on the bus.

- If its arbitration number is equal to the number on the bus then it gets the bus. 
$\bmod 0$

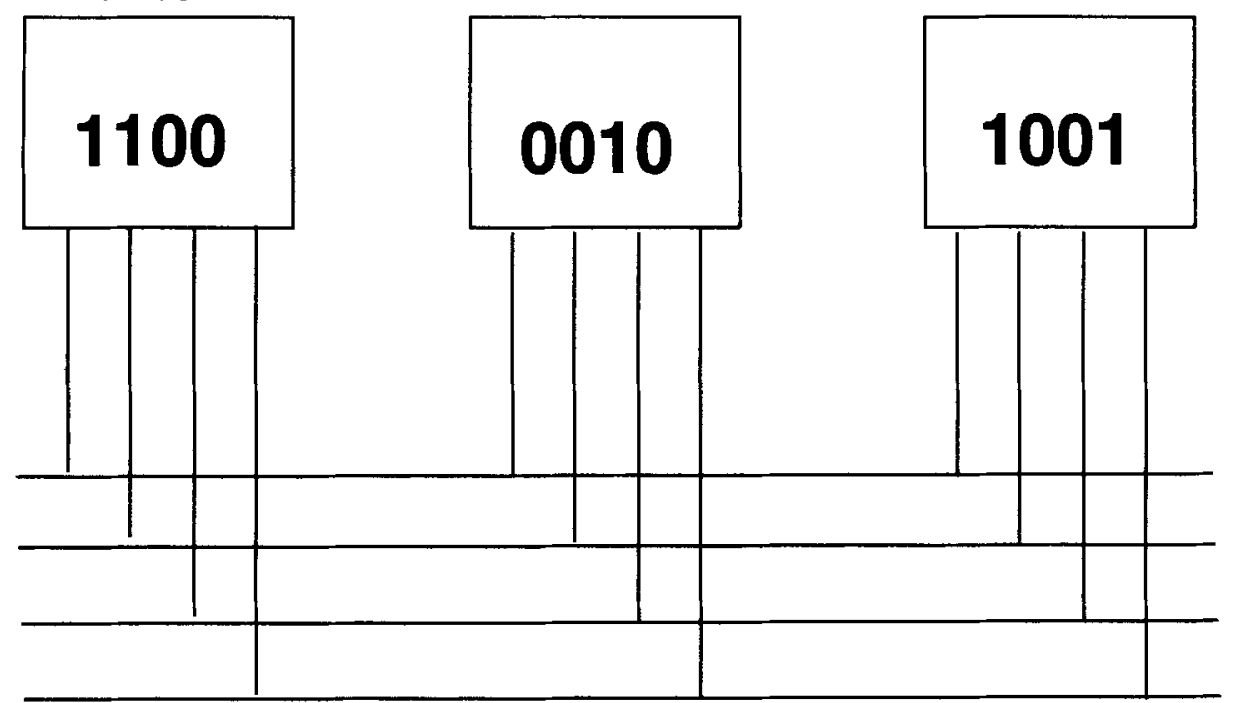

Figure 1. The system.

- If its arbitration number is not equal to it then it performs the following operation:

Let the arbitration number of the module be: $m_{1}, m_{2}, \cdots, m_{P}$ and the number on the bus be $b_{1}, b_{2}, \cdots, b_{P}$. Let $k$ be the first digit from left-to-right such that $b_{k}>m_{k}$. Then, the module puts a number corresponding to $m_{1}, \cdots, m_{k-1}, 0, \cdots, 0$ on the lines and waits indefinitely until the kth bit becomes zero and then reenters the competition.

- Each module can use the bus for a finite amount of time.

The bus performs the following operations:

- The value on the bus is equal to the bitwise "or" of the values put on the lines from all the modules.

Now, the design of the protocol can be described as follows:

Given that the arbitration numbers assigned to the modules are distinct and are not composed of just 1's or just 0's, design a protocol such that access to the bus is done in a mutually exclusive way and there is no deadlock.

To fix the ideas, consider the case where $N$ equals 3 and $P$ equals 4 . This is shown in figure 1 . The 3 modules are named mod0, mod1 and mod2 and their arbitration numbers are respectively $1100,0010,1001$. The order is the natural order $0010<1001<$ 1100 , so modI has the lowest priority and mod0 has the highest.

The behaviour of the protocol is briefed below:

1. Place the arbitration number on the lines.

2. Read bus lines in order and compare them with the arbitration number. Because of the "or" function implemented by the bus, there is no possibility of a module reading a 0 on a line if it has previously put a 1 on it. 
Now, when a module reads a 1 corresponding to $\mathrm{a} 0$ in its arbitration number, it stops to compete for a while and does not continue to read the subsequent lines. Instead, it places 0 's on all the successive lines to let others the possibility to win and remains blocked reading the same line until a 0 appears on the line on which it is blocked. This is done to avoid the blocking (or the deadlock).

3. After the module places its number on the last line without getting stuck, it tests whether its number and the number on the bus are the same. If they are the same then the module knows that it is the winner. Otherwise, it is inferred that another module has already won over it and hence, waits on the line corresponding to the position of the most significant digit position which is less than that on the corresponding bus line. To see that others are not blocked because of the possible 1's in the least significant digit positions after that position, it places a zero on all the successive lines.

\section{Synchronous solution}

In this section, we describe a solution in ESTEREL and discuss its properties. An informal briefing of the kernel statements are given in appendix A; for details the reader may refer to Berry (1992) and Berry \& Gonthier (1992).

\subsection{Solution in Esterel}

A solution in Esterel with $N=4$ and $P=3$ is described below; the generalization to any $N$ and $P$ follows naturally. The solution has the following structure: Each module has two phases: reading and checking. In the reading phase, the module places its number on the lines; in the checking phase, it checks whether its number and the number on the bus are the same. If so, it captures the bus and releases it; otherwise, it awaits for the higher priority processes to finish and lets other to win over it by placing 0 's from the point it is stuck. The actual solution in ESTEREL is given below:

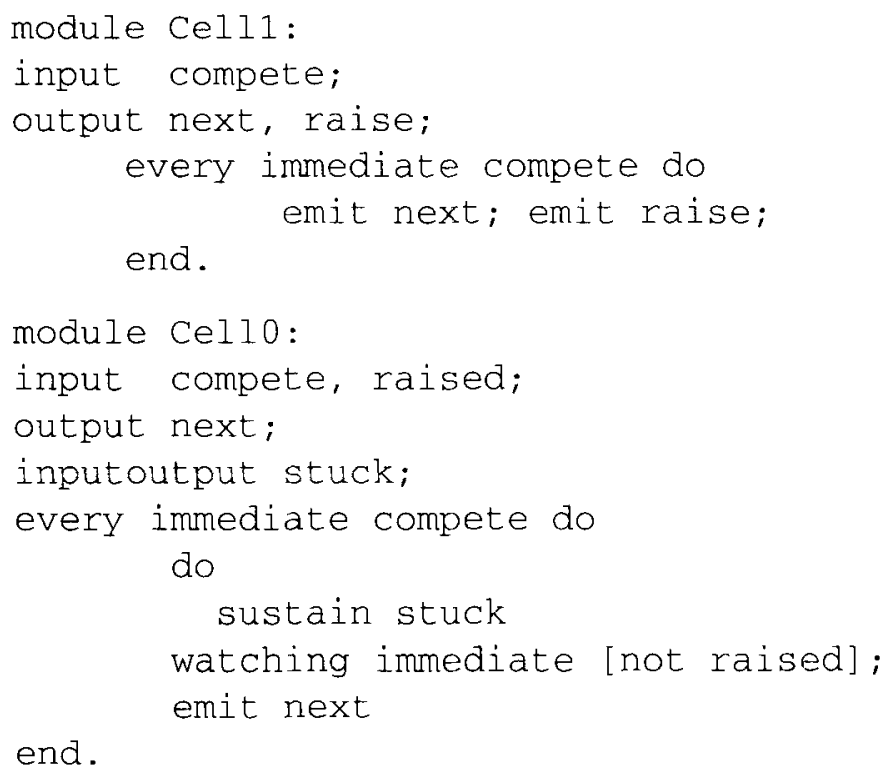


module line:

input raise;

output raised;

loop

present raise then await tick; enit raised

else await tick

end;

end.

module start_result:

input start, result;

inputoutput stuck;

output go, success;

signal 1stuck in

loop

await start; emit lstuck;

trap term in

[sustain go

II await immediate [result and not lstuck];

emit success; exit term

llevery immediate stuck do await tick; emit lstuck end ] ;

end;

end;

end.

module FUTUREBUS:

input start1, start2, start3;

output success 1 , success 2 , success 3 ;

inputoutput stuck1, stuck2, stuck3;

output raise1, raise2, raise 3 , raise4;

signal raised1, raised2, raised3, raised4, result1, result2, result3 in

signal pass1, pass2, pass3, go in run Cell1[signal go/compete, pass1/next, raise1/raise]

II run Cello[signal pass1/compete, pass2/next, raised2/raised, stuck1/stuck ]

II run Cell1[signal pass2/compete, pass3/next, raise $3 /$ raise]

$1 /$ run Cell1[signal pass3/compete, result1/next, raise4/raise] 
II run start_result[signal start1/start, success1/success, result1/result, stuck1/stuck]

end signal

| |

signal pass1, pass2, pass3, go in

run cello[signal go/compete, pass1/next, raised1/raised, stuck2/stuck]

II run Cell1[signal pass1/compete, pass2/next, raise2/raise]

I| run cel11[signal pass2/compete, pass3/next, raise3/raise]

1/ run Cello[signal pass3/compete, result2/next, raised4/raised, stuck2/stuck]

I| run start_result[signal start2/start, success $2 /$ success, result2/result, stuck2/stuck ]

end signal

| |

signal páss1, pass2, pass3, go in

run Cel11[signal go/compete, passl/next, raise1/raise]

$1 /$ run Cel10[signal pass1/compete, pass2/next, raised2/raised, stuck3/stuck ]

11 run Cel10[signal pass $2 /$ compete, pass 3/next, raised3/raised, stuck3/stuck ]

II run Ce110[signal pass3/compete, result3/next, raised4/raised, stuck3/stuck]

11 run start_result [signal start3/start, success $3 /$ success, result3/result, stuck3/stuck] end signal

| I run line[signal raised1/raised, raise1/raise]

I| run line[signal raised2/raised, raise2/raise]

| I run line[signal raised3/raised, raise3/raise]

1) run line[signal raised4/raised,raise4/raise] end.

An informal description of the program is given follows:

1. Each process is defined as a parallel composition of four cells (modules cello or cel 11 which carry zero and one respectively) and an interface process modelled as an ESTEREL module start_result. The module start_result maintains the start of modules and the success propagation. The bus is modelled as a parallel composition of four lines.

2. On receiving a compete input signal, the process initiates the competition; the first cell triggers the action of placing the numbers from the most significant position onwards till the complete number is put on the arbitration bus; all the processes wanting to take the bus do the same thing. This corresponds to the registering phase as per the specification. The reading is initiated by a compete and the next cell is triggered 
by the next signal after placing raise on the bus line if it is the cell carrying one. The completion of the registering phase is signalled by the respective result signal and the checking phase starts. In the checking phase, the process proceeds as in the registering phase till its value and the bus value are not the same; on each " 1 " it sustains the raise. Once it finds that some other process has put a " 1 " and its own value is zero then it gets stuck; as the 1's in the subsequent positions are not sustained there is no explicit need for placing 0 's. At this time it generates a stuck signal.

3. A process that is stuck can reenter registration phase in the next step when the line on which it is waiting becomes zero (i.e., not (raised)). Then the reading (for the subsequent bits) and the checking (for the whole number) repeats. If the process completes checking the line twice without getting stuck consecutively then it knows that it is the largest and takes the bus and releases it.

\subsection{Properties of the solution}

In this section, we argue that the solution satisfies the properties of mutual exclusion and deadlock-freeness. We assume that the arbitration numbers are distinct and are not composed of just 1's or just 0's.

\section{PROPOSITION 1}

The above solution guarantees mutual exclusion.

Proof. As the numbers are distinct, it can be easily seen that there is a total order on the arbitration numbers. Now, we can prove mutual exclusion of the access as follows: let us assume that at some point of time a nonempty subset of modules enter the competition to capture the bus. If the subset is a singleton set, it is obvious that the module places the number and tests its identity and captures the bus. Now, consider a subset consisting of more than 2 modules. Since, the arbitration numbers cannot be composed of only 1's at the end of the first round all the modules will be stuck. All the processes withdraw from the competition by setting 0's from the most significant digit, say $\mathrm{k}$, at which it is less than the kth bus digit. The processes can enter into competition only after the position at which they are waiting becomes zero. Because of the total order and the fact that all the blocked processes put zeros from the position of their being blocked, it should be obvious that it is only the modules with the highest number up to the kth position that can enter into competition again. It may be noted that there can be more than one module that can enter into competition. Further, it can be easily observed that if two modules get stuck at the kth position, then their prefix up to this digit must be the same exactly. Hence, if there are two modules stuck at the last digit, it means they must be identical - which contradicts the assumption of being distinct numbers. In the next round, in addition to these modules wanting to reenter the competition, there can be new modules entering into competition. In this round, the modules which are less than those that are blocked so far (up to the kth digit) will get blocked by the existing modules and those that are greater can progress. After reentering the competition, the blocked processes only put their numbers from the $k+1$ position to the nth position. After the modules have put their numbers they check 
for their complete arbitration number against the number on the bus. As the numbers are distinct there can be only one winner and hence, the mutual exclusion.

\section{PROPOSITION 2}

The solution is deadlock free.

Proof. From the proof of mutual exclusion, we can conclude that it is the module having the highest number that wins the competition. Since the numbers form a total order, it is obvious that a winner will be chosen in at most $N$ instants (each digit can block one instant). Hence, the proof follows.

\section{PROPOSITION 3}

The solution is not livelock free assuming there are at least three modules.

Proof. Consider three modules $M_{1}, M_{2}, M_{3}$ having arbitration numbers

$$
1 a_{2} \cdots a_{n}, 1 b_{2} \cdots b_{n} \text { and } 0 c_{2} \cdots c_{n}
$$

respectively and let us assume that the numbers are distinct are not composed of just 1's or 0's as discussed earlier. Consider the following scenario:

$M_{1}$ is already using the bus and $M_{3}$ is waiting on the first bit. Then, before $M_{1}$ finishes $M_{2}$ can get into competition and overshoot $M_{3}$; now $M_{1}$ and $M_{2}$ can conspire to see that $M_{3}$ never gets its turn.

The solution is not livelock-free follows from the above scenario.

Note: In the case when there are only two modules, assuming that the releasing process gives way to the process waiting on it, there is no possibility of a livelock.

\subsection{Solution with Explicit Master}

By introducing a sentinel value on the bus and another registering phase, we can obtain a solution where one distinguishes the master and the master elect (i.e., the one which becomes the master as soon as the current master relinquishes the resource). The solution is given in the following. It must be noted that in the context of preemption there is no clear identity of Master Elect as it can be changed before the master releases the main bus.

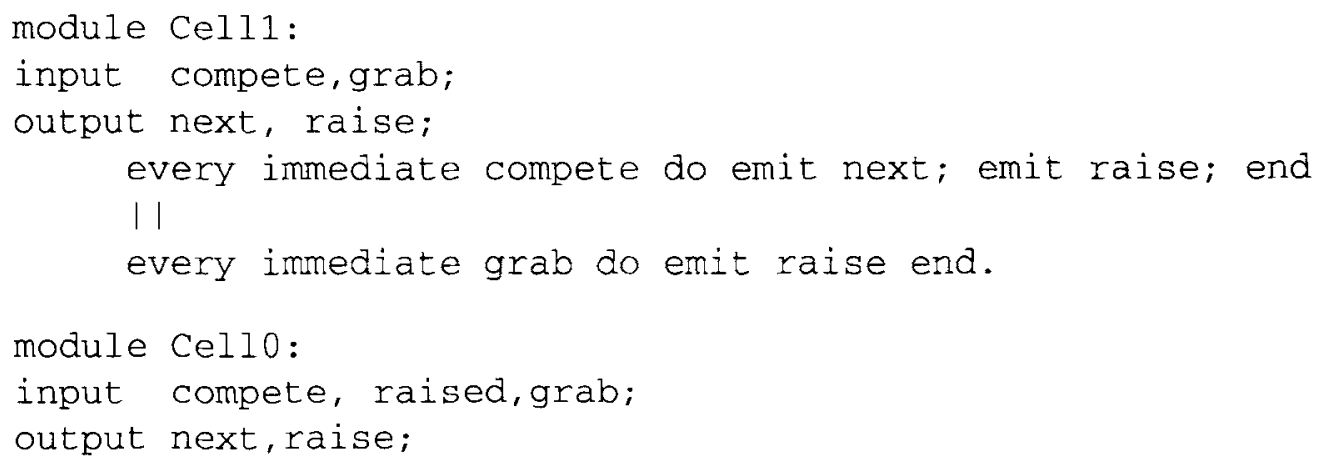


inputoutput stuck;

every immediate compete do

do

sustain stuck

watching immediate [not raised];

emit next

end

| |

every immediate grab do emit raise end.

module line:

input raise;

output raised;

loop

present raise then await tick; emit raised else await tick end

end.

module start_result:

input start, result;

inputoutput stuck;

output go, success, grab;

signal lstuck in

loop

await start; emit lstuck;

trap term in

[sustain go

I I await immediate [result and not lstuck];

await tick; present result then emit success;

exit term end

l levery immediate stuck do await tick;emit lstuck end ] end;

\% The process reads the bus twice before declaring success.

\% The watching statement used below enables the process to

o grab the line in three steps. The grab makes all the

\% cells to raise the line.

do

sustain grab

watching 3 tick;

end;

end.

module FUTUREBUS:

input start1, start2, start3;

output success1, success 2, success 3 ;

inputoutput stuck1, stuck2, stuck3; 
output raise1, raise2, raise3, raise4;

signal raised1, raised2, raised3, raised4, result1, result2, result3, grab1, grab2, grab3 in

signal pass1, pass2, pass3, go in

run Cel11[signal go/compete, passl/next,

raise1/raise, grab1/grab]

I I run Cel10[signal pass1/compete, pass2/next, raised2/raised, raise1/raise, stuck1/stuck, grab1/grab]

II run Cell1[signal pass2/compete,pass3/next, raise3/raise, grab1/grab]

1) run Celli[signal pass3/compete, result1/next, raise4/raise,grab1/grab]

I| run start_result[signal start1/start, success1/success, result1/result, stuck1/stuck,grabl/grab] end signal

|

signal pass1, pass2, pass3, go in

run Cel10[signal go/compete,passl/next, raised1/raised, raise1/raise, stuck2/stuck, grab2/grab]

1/ run Cel11[signal pass1/compete,pass2/next, raise2/raise,grab2/grab]

I| run Cel11[signal pass2/compete, pass3/next, raise3/raise, grab2/grab]

|| run Cello[signal pass3/compete, result2/next, raised4/raised, raise4/raise, stuck2/stuck,grab2/grab] II run start_result[signal start2/start, success2/success, end signal | | result2/result, stuck2/stuck, grab2/grab]

signa1 pass1, pass2, pass3, go in

run Cell1[signal go/compete, pass1/next, raise1/raise, grab3/grab]

II run Cel10[signal pass1/compete, pass2/next, raised2/raised, raise2/raise, stuck3/stuck,grab3/grab]

| I run Cel10[signal pass2/compete,pass3/next, raised3/raised, raise3/raise, stuck3/stuck,grab3/grab]

| I run Cel10[signal pass3/compete, result3/next, raised4/raised, raise4/raise, stuck3/stuck,grab3/grab] |) run start_result[signal start3/start, success3/success, 


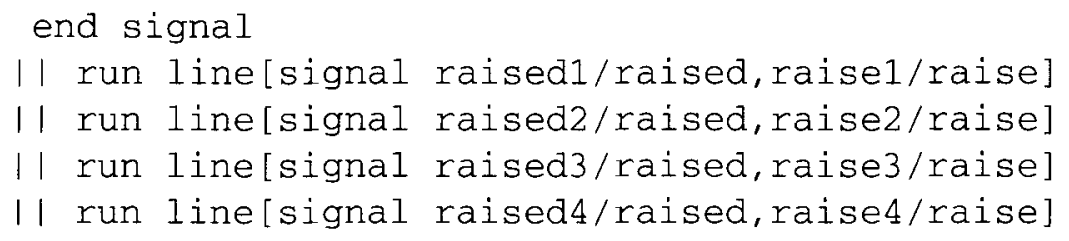

\section{Asynchronous solution}

The synchrony hypothesis of the synchronous languages leads to the assumption that whenever the modules (or processes) are ready to take an action (read/emitting) they will do it in the same instant. However, such an assumption does not hold in the asynchronous solution. In other words, we cannot guarantee that the processes enabled on some actions will really do it at that time. In other words, "," "semicolon operator cannot be just compiled away; it may correspond to different delays corresponding to different implementations.

First, we describe a high-level solution in terms of $\mathrm{C}$-code. Modules correspond to the C-functions shown in figure 2 .

The following questions will naturally arise in the reader of the above protocol;

1. What is the need of TryTakeBus? Why not take the bus immediately?

2. What is the need of resetting lines after the competition?

3. What is the need for a global test of the bus ? Is it possible to implement the same as a loop that tests for lines one after the other?

4. Is a fairness condition assumed somewhere?

5. Are all arbitration numbers allowed?

6. What confidence can we have in such a program ? Can we test or verify it?

We discuss possible answers to the above questions below.

Question 1. Modules can compete in accessing the bus at any time. Suppose mod1 reads the last line with the good value say, 0 . This means that each time it has read a line, no other module with higher priority has already changed this line. This does not mean that there is no module with higher priority competing for the bus! This could be the case if, say, mod 0 enters the competition at the last moment. The global testing of the bus rejects this situation.

Question 2. Resetting lines after working allows others modules with lower priorities to take the bus.

Question 3. The answer to the first question shows that the test of the bus must be global, that is atomic. There is no possibility in the general case, to avoid the use of a global test while respecting priorities. 


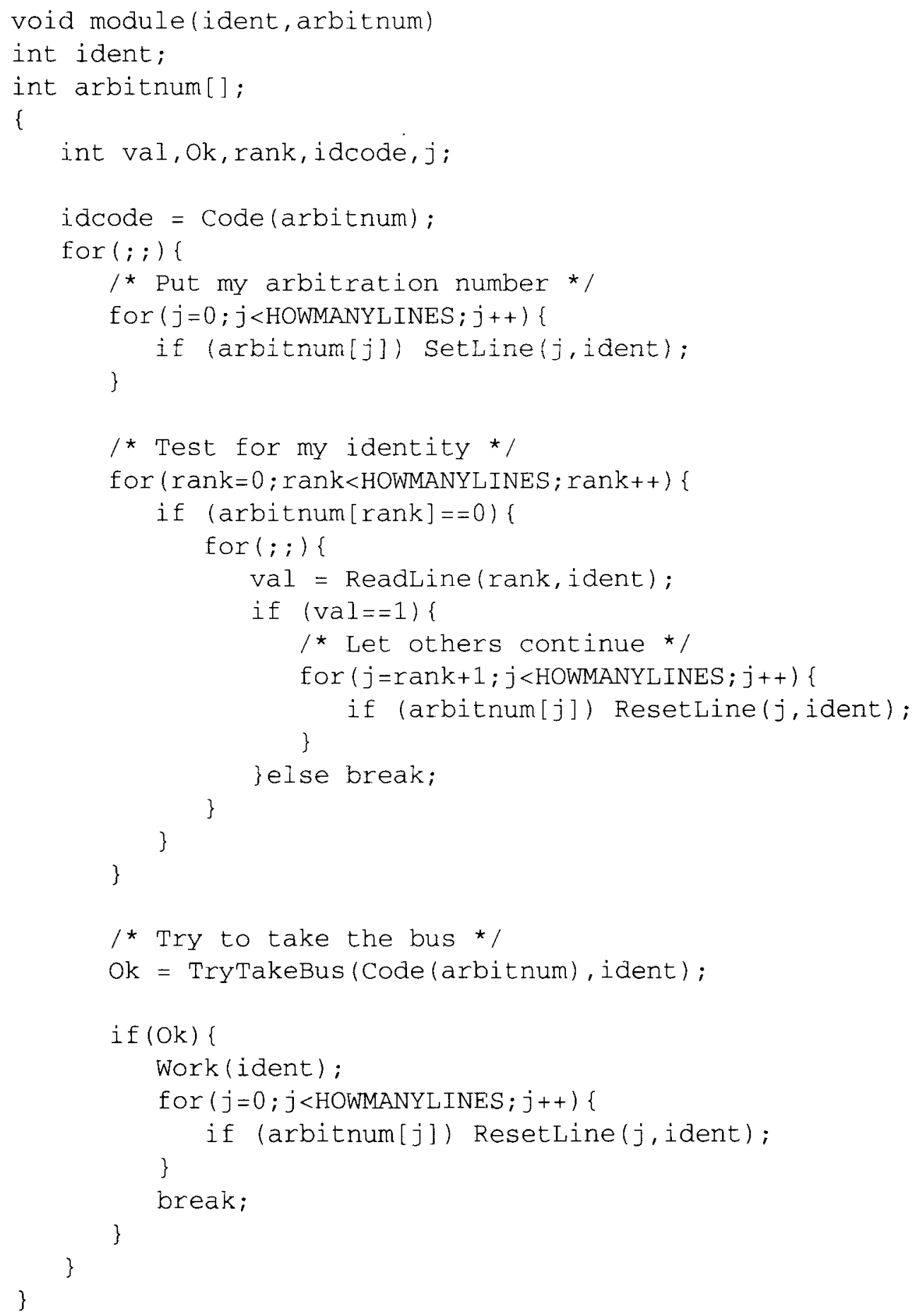

Figure 2. The protocol in $\mathrm{C}$. 
Question 4. There are possibilities of "livelocks": consider for example two modules m0 and $\mathrm{m} 1$ with arbitration numbers 01 and 10 . Consider the following possible interleaving:

- mo puts $0 ; \mathrm{m} 0$ puts $1 ; \mathrm{m} 0$ reads 0 ;

- m1 puts 1;

- mo reads $1 ; \mathrm{mo}$ tries to take the bus and fails (the value of the bus is 11 ).

- $\mathrm{m} 1$ puts $0 ; \mathrm{m} 1$ tries to take the bus and fails (the value of the bus is 11).

This behaviour can be done forever, producing a livelock.

Question 5. A quick analysis shows that not all arbitration numbers are allowed. For example consider two modules $\mathrm{m} 0$ and $\mathrm{m} 1$ with arbitration numbers 0 and 1 . The following interleaving is possible: $\mathrm{m} 0$ puts a 0 , reads the bus and sees a 0 . Thus it can begin to work. But now, 1 puts a 1 and reads a 1 , so it can also begin to work. The access to the bus is thus incorrect as two modules are working at the same time.

Question 6. Obviously, answers to the previous questions imply that we cannot have any confidence in the protocol as stated presently.

To face this problem, we are going to:

- Characterize the arbitration numbers allowed.

- Test the protocol by executing the code both in an asynchronous and in a synchronous way.

- Give a formal specification of the protocol in Meije and analyze it with the verification tools auto and autograph. We will get a correctness proof of the protocol for the case $N=P=3$, with 3 fixed arbitration numbers.

\subsection{Arbitration numbers allowed}

Consider condition $\mathrm{C} 1$ : any two arbitration numbers are bitwise incomparable. For example, 01 and 10 verify $\mathrm{C} 1$. On the contrary, 11 and 10 do no verify $\mathrm{C} 1$.

We have the following property: Correct access to the bus is equivalent to $\mathrm{C} 1$.

Proof. Suppose a wrong access to the bus by two modules $\mathrm{m} 0$ and $\mathrm{m} 1$. This means that one module, say mo, has taken the bus by seeing its arbitration number $\mathrm{A} 0$ on the bus, while $\mathrm{m} 1$ is also in competition and has already put some 1 values. As m1 also takes the bus, all 1 's in A0 must also be present in A1. So A1 is comparable to A1. Conversely, suppose that $\mathrm{A} 0$ and $\mathrm{A} 1$ are not comparable and one module, say mo, first takes the bus. Then $\mathrm{m} 1$ cannot also take it as it will never see its arbitration number on it.

Now, let us consider priorities. Respect of priorities means that a module with lower priority cannot win if a module with higher priority is also in competition. So respect of priorities depends on the definition of being in competition.

There are two distinct approaches:

1. A module enters the competition after it has put the first 1 . Call this "competition from the first". 
2. A module enters the competition after it has put the last 1 . Call this "competition from the last".

We have the following properties:

1. For competitions from the last, $\mathrm{Cl}$ implies respect of priorities.

2. This is not the case for competitions from the first.

Proof. Suppose a situation where two modules $m 0$ and $m 1$ compete for the bus and $m 0$ wins although it has a lower arbitration number. Then all the 1 's are already put by $\mathrm{m} 1$ (because we are considering competition from the last) and they necessarily correspond to 1 put by mo (otherwise mo could not have taken the bus). So $\mathrm{m} 1$ 's arbitration number is less than that of mo, which violates the hypothesis.

For the second property, consider $\mathrm{mo}$ and $\mathrm{m} 1$ with 110 and 101. These two arbitration numbers verify $\mathrm{C} 1$. Now consider the following interleaving: $\mathrm{m} 0$ puts 1 , then $\mathrm{m} 1$ puts 1 , then 0 , then 1 , then reads its arbitration number on the bus and takes it. This scenario does not respect priorities as mo has higher priority than $\mathrm{ml}$.

Now let us define the condition C2 by: if two arbitration numbers have a 1 at the same place, they necessarily differ before this place. Examples: 110 and 101 do not verify $\mathrm{C} 2$. On the contrary, 110 and 011 do.

We have the property: For competitions from the last, $\mathrm{C} 2$ implies respect of priorities.

\subsection{The asynchronous version}

We define the bus as a reactive process that is used by modules.

The bus The bus is implemented as an array, with $\mathrm{C}$ functions to use it. The $\mathrm{C}$ code is the following:

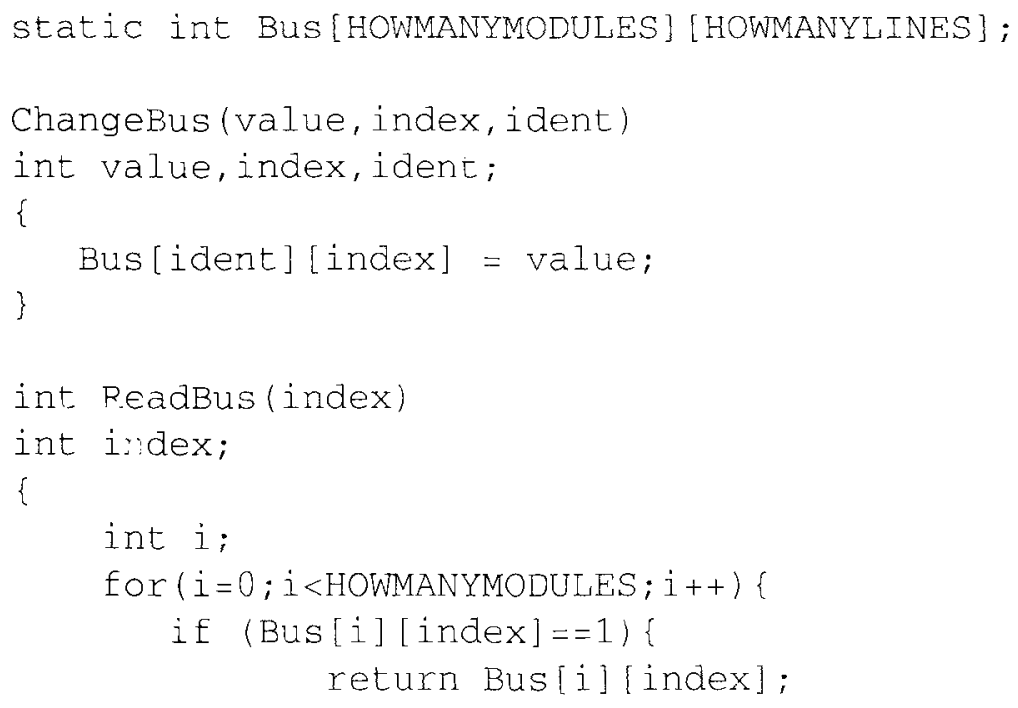




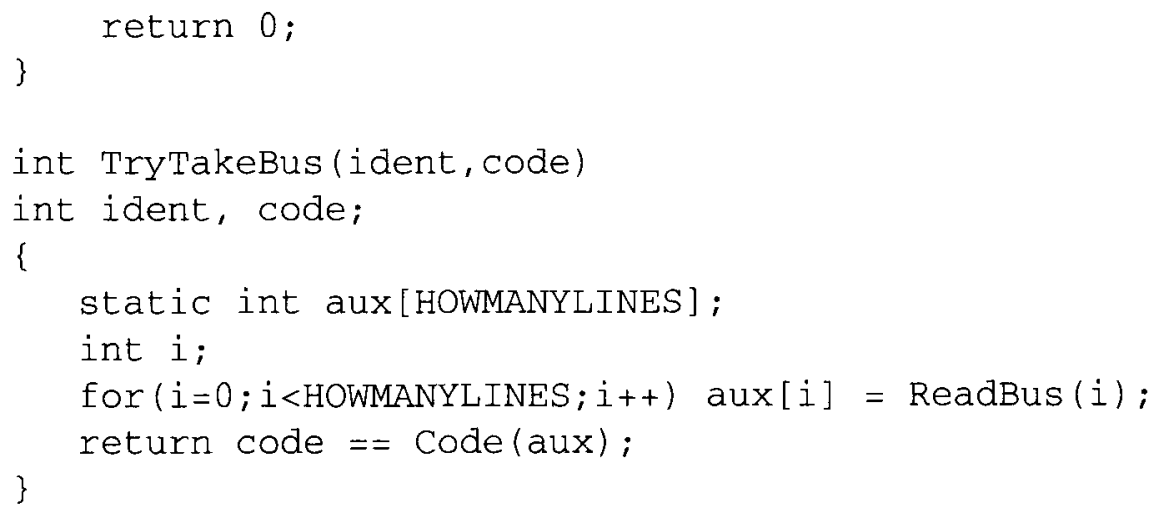

We define a reactive process that implements the bus in the following way:

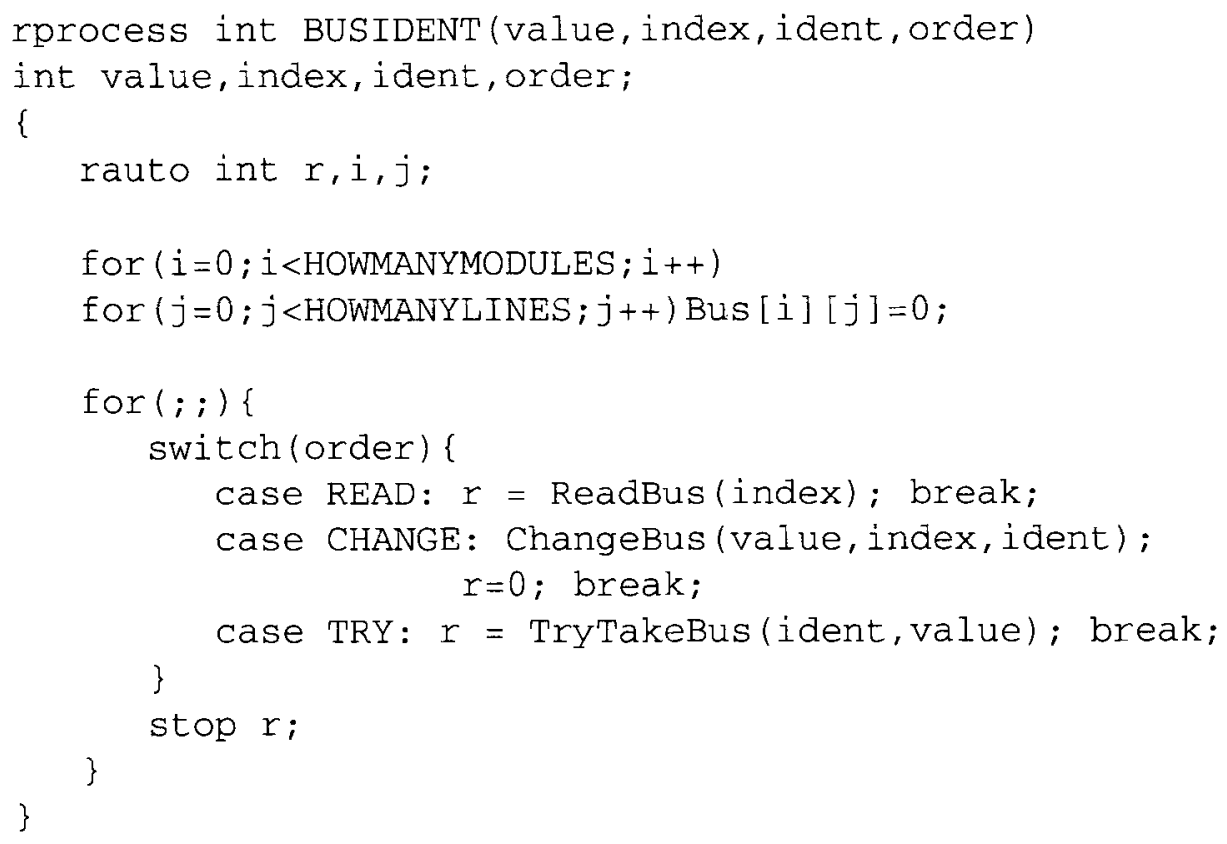

Modules Modules are using the bus as a task:

rtask int Bus (value, index, ident, order)

int value, index, ident, order;

\{ rprocess BUSIDENT MachineForBus; \}

To get one reaction of the bus, we have the following code:

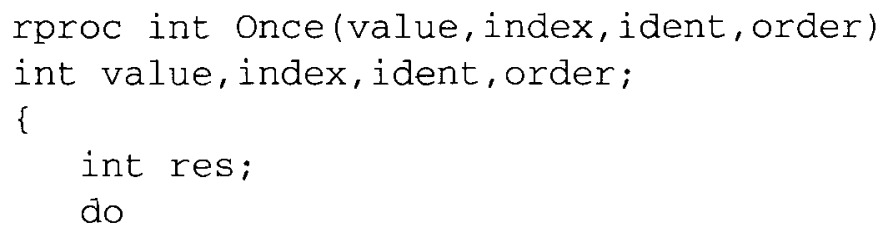




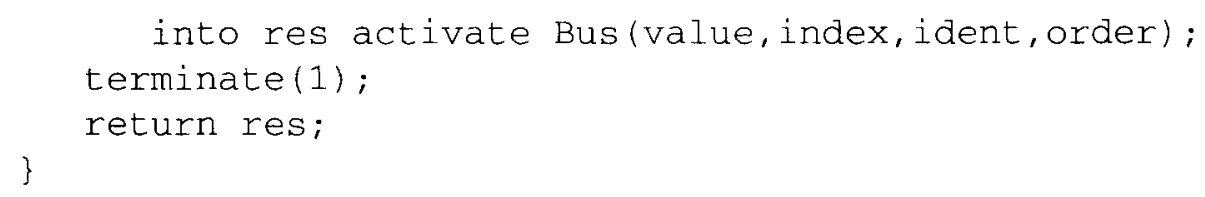

The code for a module is very similar to the $\mathrm{C}$ code given previously. In fact, the only change is in the use of the bus through reactive procedures instead of $\mathrm{C}$ functions. We also declare variables as rauto to retain their values from one instant to the next and enclose the code in a loop to limit the participation to the competition. A module corresponds to the following RC code:

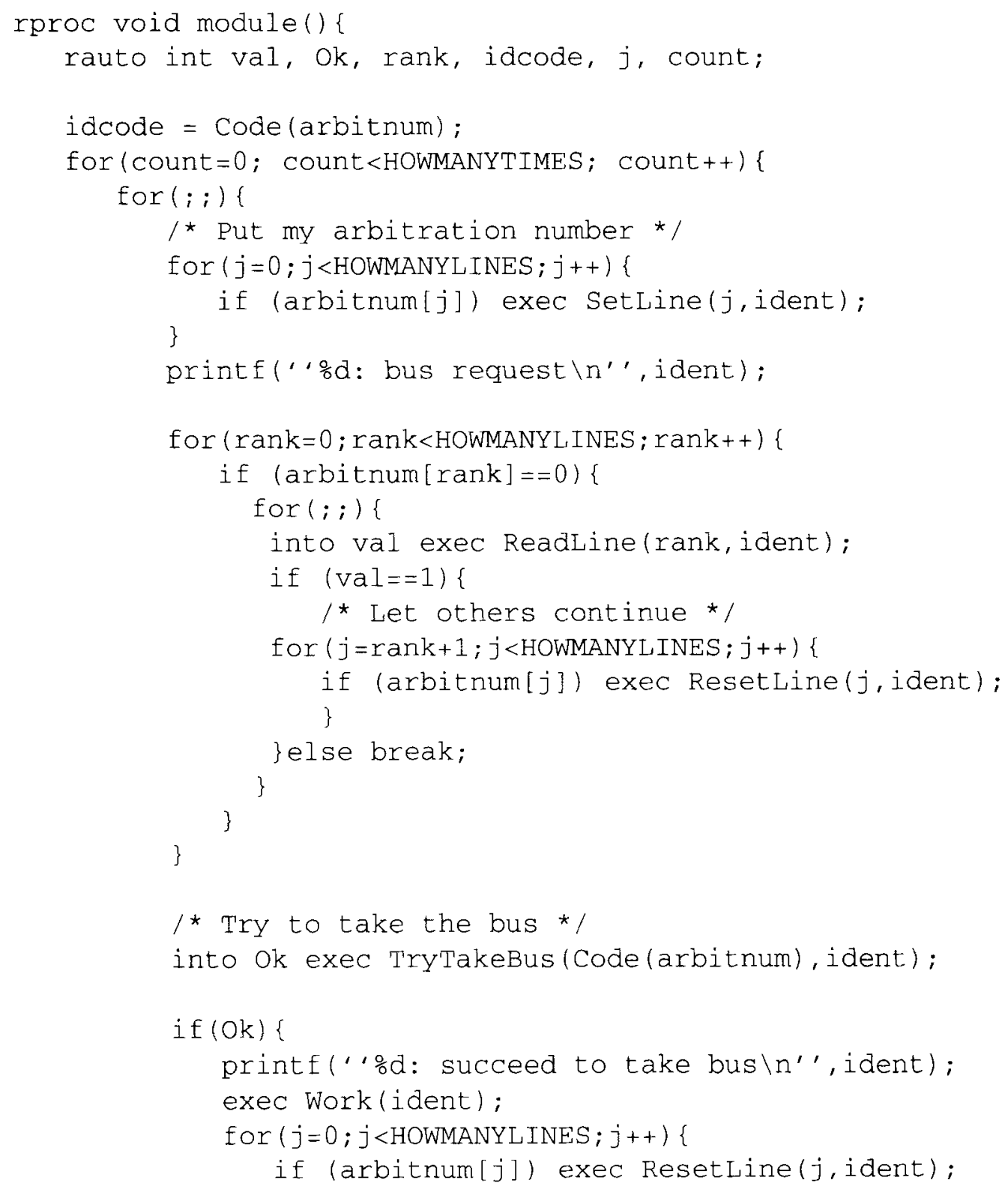




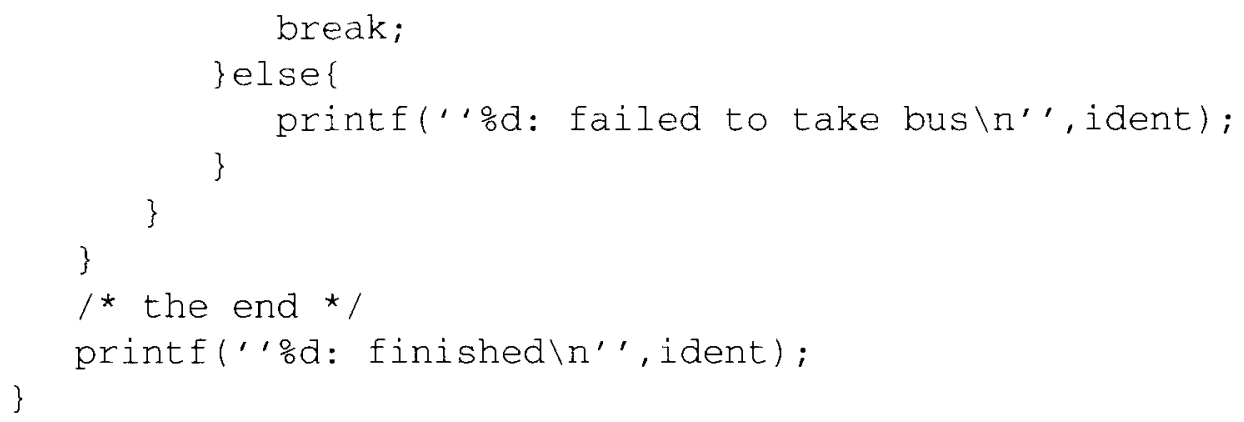

Notice that competition from the last is assumed as "bus request" is printed after the last 1 has been put on the bus.

By running this code on several machines, we can test the protocol in asynchronous contexts. For example, we can run the bus on the cma machine and two modules on the same dance machine and get the following result:

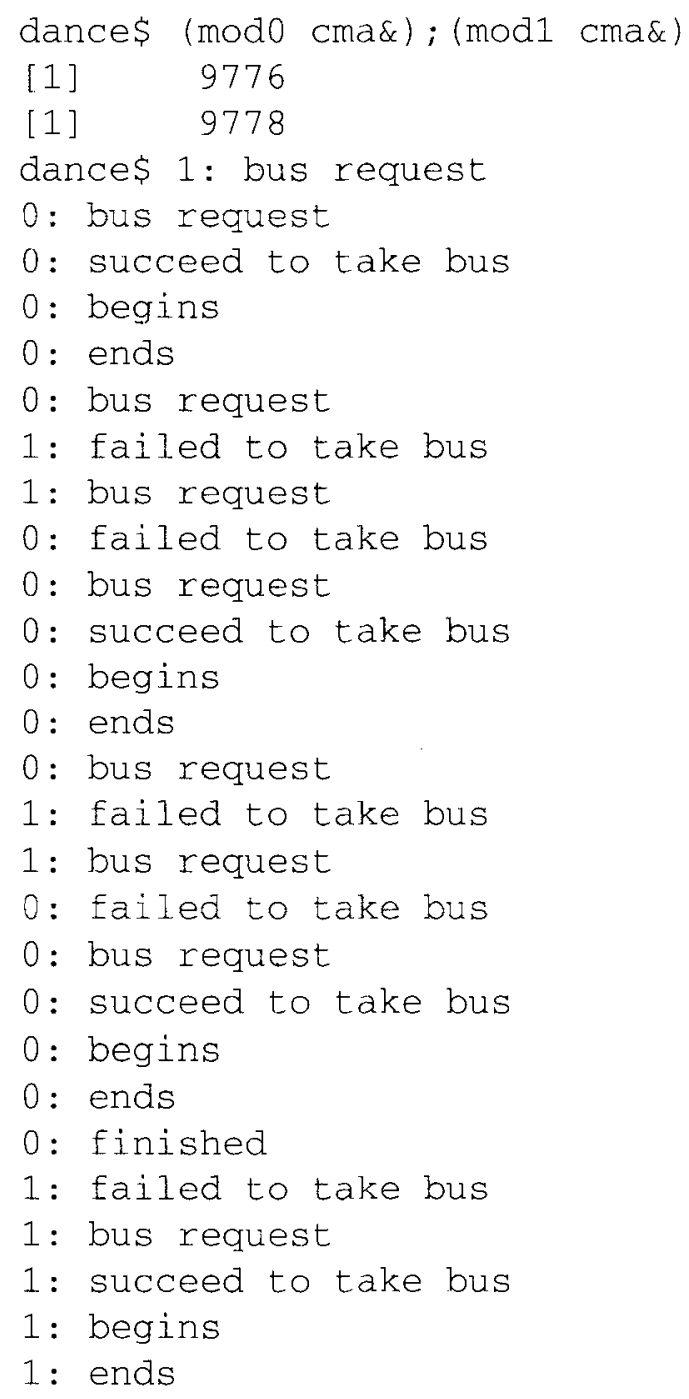




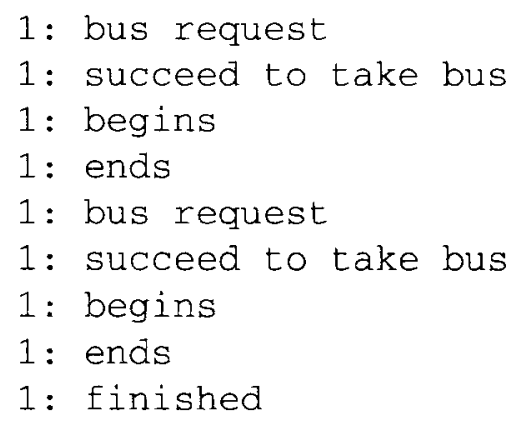

\section{Validations of the protocol}

In this section, we analyze the protocol in a particular case where $N=P=3$, and with 3 arbitration numbers 100,010 and 001 . We model lines, modules and the global system by three terms of the Meije process calculus. The main feature of Meije is the possibility to write combined actions. This feature is used to model the global test of the bus. This could not be possible using a calculus that does not allow for combined actions, such as CCS.

The line is represented by the following term that is shown on figure 3 .

The line

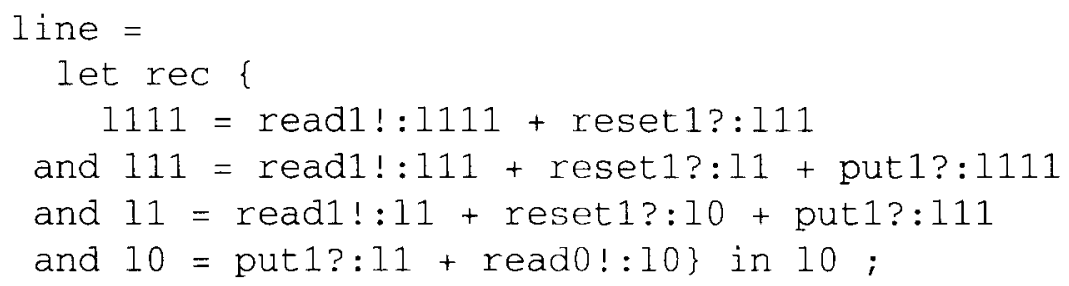

The module We have implemented the global test of the bus by the composite action "read1i?.read2j?.read3k?. success!". We have 3 modules almost identical. For example, the module with arbitration number 100 is represented by the following term that is shown on figure 4 . It consists of 3 cells, one for each line, and in addition, of a fourth component to check for success.

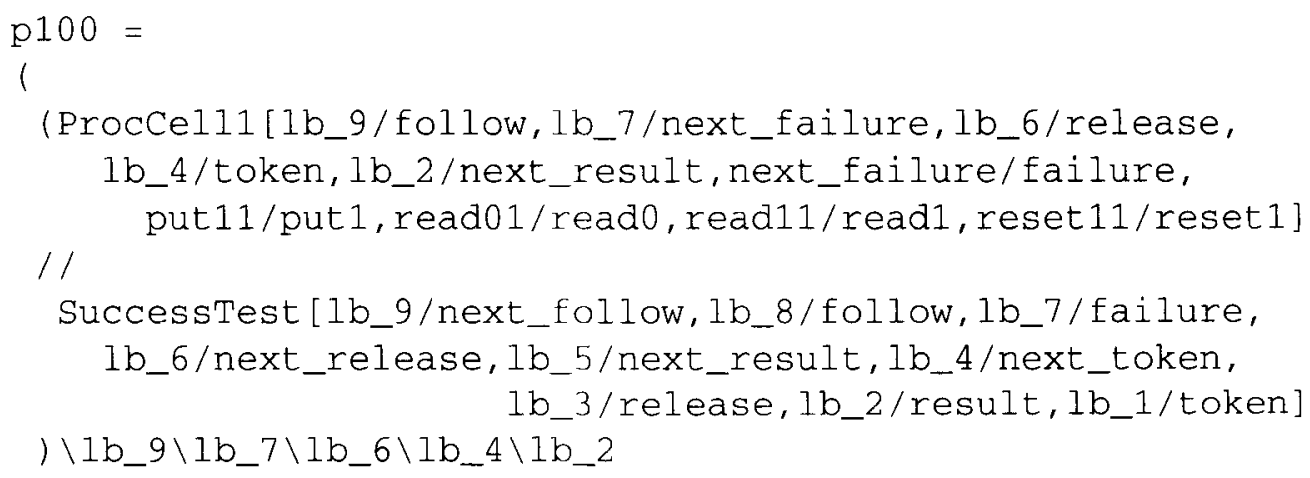




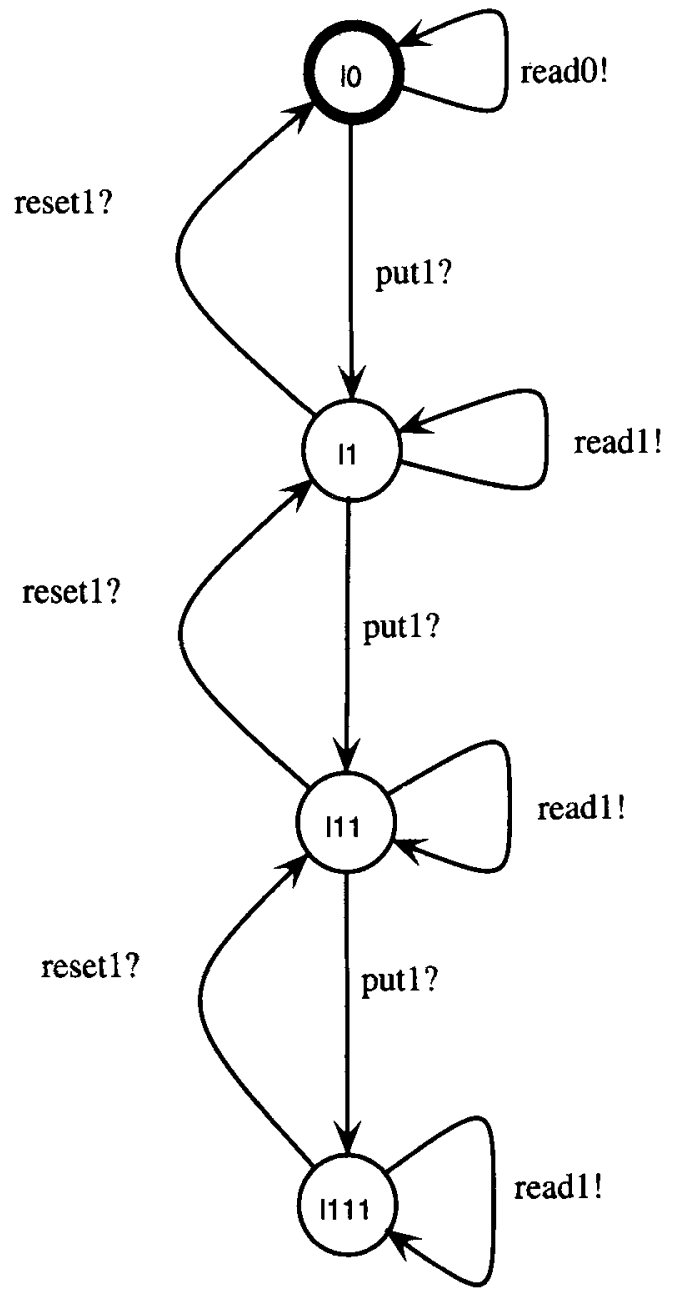

Figure 3. The line.

\section{1}

(ProcCel10[1b_0/failure, follow/next_follow, next_result/result, release/next_release, token/next_token,

next_follow/follow, result/next_result,put 12/put1, read02/read0, read 12 /read1, next_release/release, reset $12 /$ reset 1 , next_token/token] 


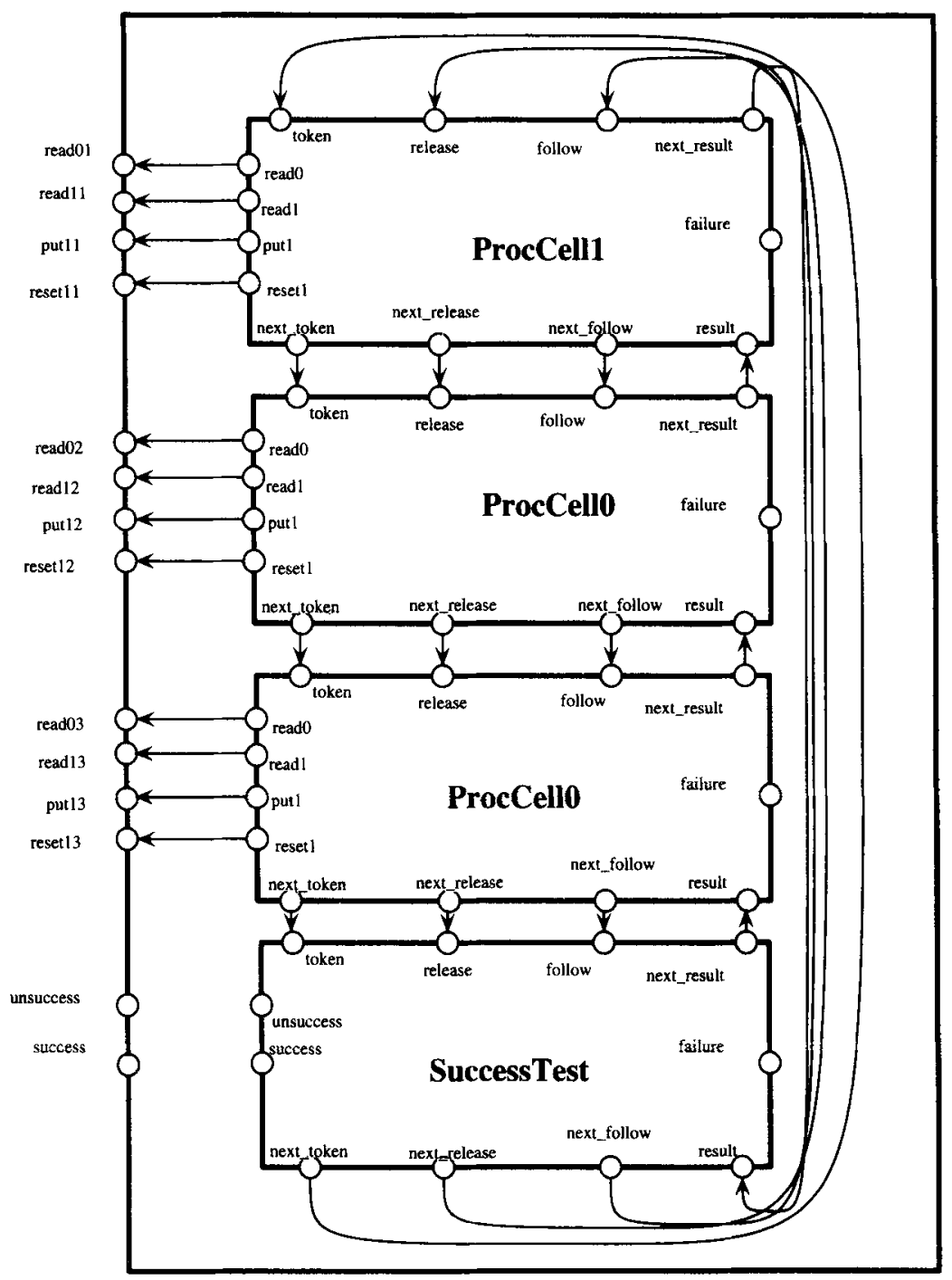

Figure 4. The module 100.

1b_1/next_token,1b_0/next_failure,put13/put1, $\mathrm{read} 03 / \mathrm{read} 0, \mathrm{read} 13 / \mathrm{read} 1, \mathrm{reset} 13 / \mathrm{reset} 1 \mathrm{]}$ )$\backslash 1 b \_0 \backslash f o l l o w \backslash f a i l u r e \backslash$ next_result $\backslash r e l e a s e \backslash t o k e n$

)$\backslash 1 \mathrm{~b} \_8 \backslash 1 \mathrm{~b} \_5 \backslash 1 \mathrm{~b} \_3 \backslash 1 \mathrm{~b} \_1 \backslash$ next_failure $\backslash$ next_follow \result \next_release \next_token;

The cells can be of two types: ProcCell1 or ProcCello corresponding to:

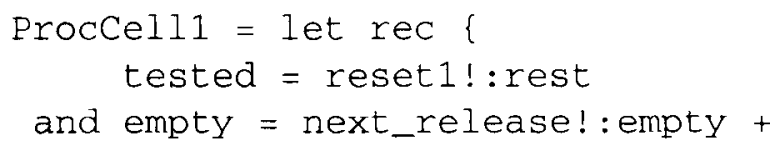




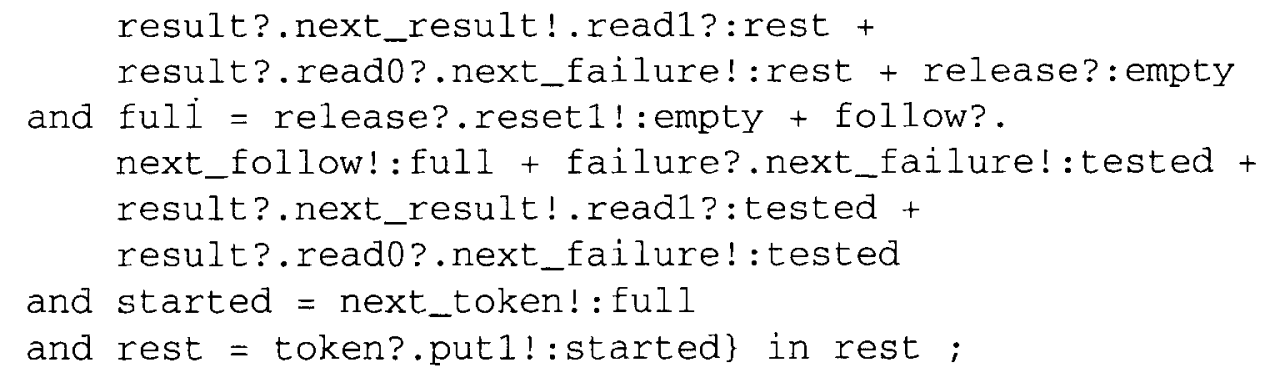

and:

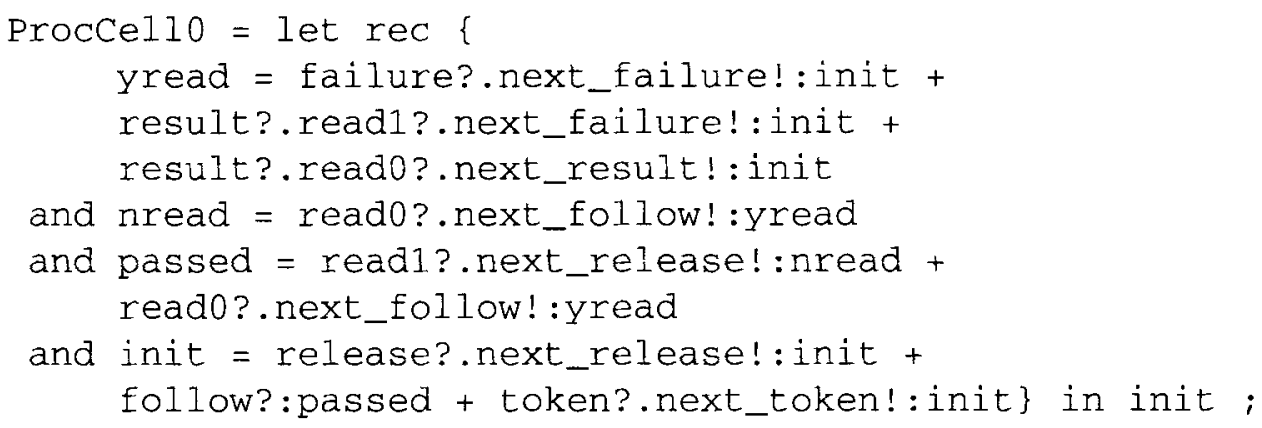

The system In the system, we put in parallel 3 modules and 3 lines. All actions except success are hidden as can be seen by the parse term given below.

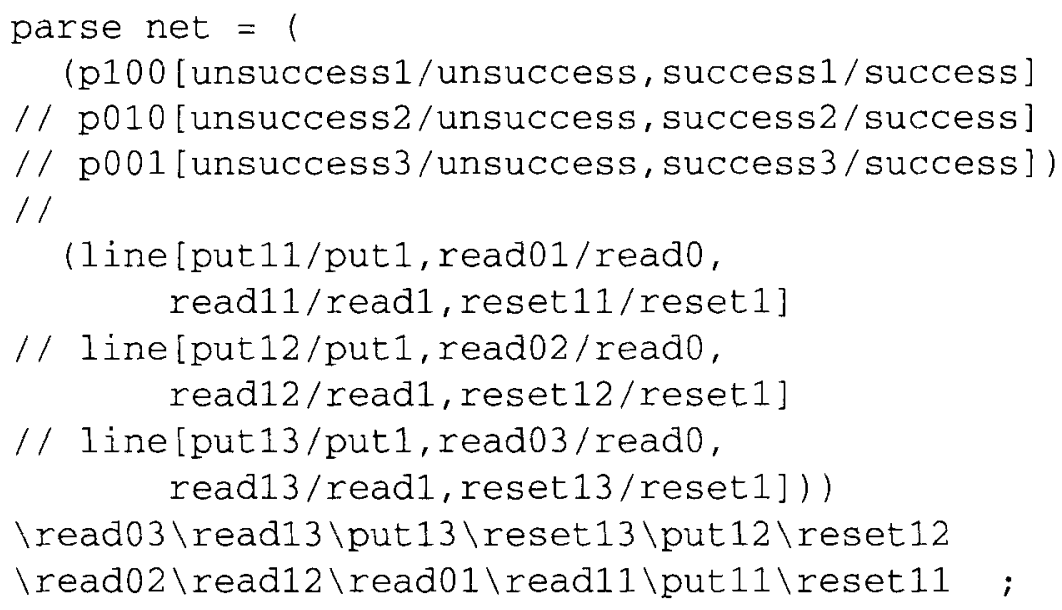

Using AUTO, it is possible to show the observational automata indeed has the behaviour required.

\section{Conclusions}

In the previous sections, we have described synchronous and asynchronous solutions for the Futurebus arbitration protocol. Our analysis reflect that ESTEREL or RC-language frameworks provide a smooth framework for modelling, testing, validating and verifying 
the solutions. Further experimentation is necessary in this direction. In fact, in Clarke et al (1992), hardware description languages have been used for finding the discrepancies in the Futurebus Cache Protocols; we have also effectively used ESTEREL for the same purpose with the same findings. To sum up,

- A synchronous solution is useful even for the distributed case. It gives the possibility of making tests by having control on interleavings. The advantage is that bugs can be reproduced and analyzed.

- There must be a smooth transition between synchronous and asynchronous solutions. Hence, there is a need for a unified framework for asynchrony and synchrony. In this paper, we have used RC for coding and testing the synchronous and asynchronous versions with minimal changes. Currently, we are trying to use the recently proposed unified framework, CRP (Berry et al 1993; Shyamasundar 1993) for these aspects.

- Formal and automated proofs can be done in the above frameworks. In the above paper, we have shown the formal correctness of the protocol using auto/autograph.

This work leads us to think that presently distributed protocol design and coding needs an heterogeneous approach to combine various techniques such as proofs made by hand, asynchronous and synchronous coding, use of process algebras, automata visualization and analysis with automated tools.

The work was partially supported by IFCPAR, Indo-French Center for the Promotion of Advanced Research, New Delhi, India.

\section{References}

Berry G 1992 A hardware implementation of pure Esterel. Sadhana 17: 95-139

Berry G, Gonthier G 1992 The Esterel synchronous programming language: Design, semantics, Implementation, SCP, 19(2): 87-152

Berry G, Ramesh S, Shyamasundar R K 1993 Communicating Reactive Processes, 20th ACM POPL, South Carolina, pp. 85-99

Boussinot F 1991 An extension to C to program reactive systems. Software practice experience 21: $401-428$

Clarke E, Grumberg O, Hirashi H, Jha S, Dong D, McMillan L, Ness L 1992 Verification of the Futurebus+ Cache Protocol. CMU-CS-92-206, Pittsburgh

Le Guernic P, Le Botgne K, Gauthier T, Le Maire C C 1991 Programming real-time applications in signal. Proc. IEEE (Spec. issue) 79: 1321-1336

Halbwachs N, Caspi P, Pilaud D 1991 The synchronous dataflow programming language Lustre Proc. IEEE (Spec. issue) 79: 1305-1320

Harel D 1987 A visual approach to complex systems. Sci. Comput. Program. 8: 231-274

IEEE Futurebus+: Logic Laver Specifications 1991 Draft 8.2 (IEEE Computer Society Press)

Roy V, de Simone R 1990 Auto and autograph. In Proc. Workshop on Computer Aided Verification, New-Brunswick, (ed.) R Kurshan 
Shyamasundar R K 1993 Specification of hybrid systems. In CRP, Proc. of AMAST 93, Workshops. Computing Series (eds) M Nivat, C Rattray, T Rus, G Scollo (Berlin: Springer-Verlag) pp. 227 238

\section{Appendix A: Esterel language}

The basic object of Esterel without value passing, referred to as PURE Esterel, is the signal. Signals are used for communication with the environment as well as for internal communication.

The programming unit is the module. A module has an interface that defines its input and output signals and a body that is an executable statement:

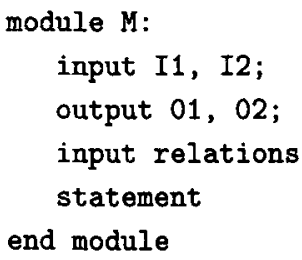

Input relations can be used to restrict input events (Berry 1992). We shall only use exclusions, written in the interface part as

relation I1 \# I2;

Such a relation that input events cannot contain I1 and I2 together. It is therefore an assertion on the behavior of the asynchronous environment.

At execution time, a module is activated by repeatedly giving it an input event consisting of a possibly empty set of input signals assumed to be present and satisfying the input relations. The module reacts by executing its body and outputs the emitted output signals. We assume that the reaction is instantaneous or perfectly synchronous in the sense that the outputs are produced in no time. Hence, all necessary computations are also done in no time. In PURE Esterel, these computations are either signal emissions or control transmissions between statements; in full Esterel, they can be value computations and variable updates as well. The only statements that consume time are the ones explicitly requested to do so. The reaction is also required to be deterministic: for any state of the program and any input event, there is exactly one possible output event. In perfectly synchronous languages, a reaction is also called an instant.

\section{A.1 Statements}

Esterel has two kinds of statements: the primitive or kernel statements, and the derived statements that can be expanded into primitive ones by macro-expansion and make the language more user-friendly. Derived statements are not semantically meaningful and will not be presented here. The list of kernel statements is:

nothing

halt

emit $S$ 


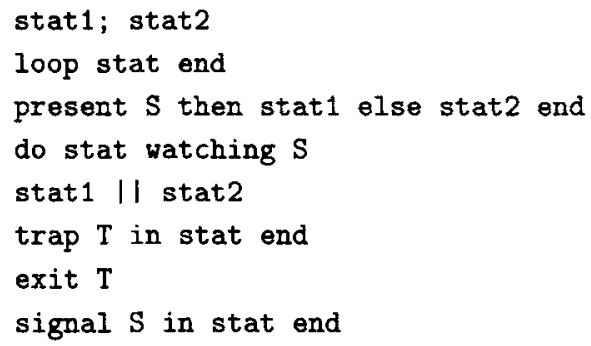

The kernel statements are imperative in nature, and most of them are classical in appearance. The trap-exit constructs form an exception mechanism fully compatible with parallelism. Traps are lexically scoped. The local signal declaration "signal in stat end" declares a lexically scoped signal $S$ that can be used for internal broadcast communication within stat. The then and el se parts are optional in a present statement. If omitted, they are supposed to be nothing.

\section{A.2 Intuitive semantics}

At each instant, each interface or local signal is consistently seen as present or absent by all statement, ensuring determinism. By default, signals are absent; a signal is present if and only if it is an input signal emitted by the environment or a signal internally broadcast by executing an emit statement.

To explain how control propagates, it is better to first give examples using the simplest derived statement that takes time: the waiting statement "await S", whose kernel expansion "do halt watching S" will be explained in a moment. When it starts executing, this statement simply retains the control up to the first future instant where $S$ is present. If such an instant exists, the await statement terminates immediately; that is the control is released instantaneously; If no such instant exists, then the await statements waits forever and never terminates. If two await statements are put in sequence, as in "await S1; await S2", one just waits for S1 and S2 in sequence: control transmission by the sequencing operator ';' takes no time by itself. In the parallel construct "await S1 || await S2", both await statements are started simultaneously right away when the parallel construct is started. The parallel statement terminates exactly when its two branches are terminated, i.e. when the last of S1 and S2 occurs. Again, the "Il" operator takes no time by itself.

Instantaneous control transmission appears everywhere. The nothing statement is purely transparent: it terminates immediately when started. An "emit S" statement is instantaneous: it broadcasts $S$ and terminates right away, making the emission of $S$ transient. In "emit S1; emit S2", the signals S1 and S2 are emitted simultaneously. In a signalpresence test such as "present $S$. ..", the presence of $S$ is tested for right away and the then or el se branch is immediately started accordingly. In a "loop stat end" statement, the body stat starts immediately when the loop statement starts, and whenever stat terminates it is instantaneously restarted afresh (to avoid infinite instantaneous looping, the body of a loop is required not to terminate instantaneously when started).

The watching and trap-exit statements deal with behavior preemption, which is the most important feature of Esterel. 
In the watchdog statement "do state watching S", the statement stat is executed normally up to proper termination or up to future occurrence of the signal $S$, which is called the guard. If stat terminates strictly before S occurs, so does the whole watching statement; then the guard has no action. Otherwise, the occurrence of s provokes immediate preemption of the body stat and immediate termination of the whole watching statement. Consider for example the statement

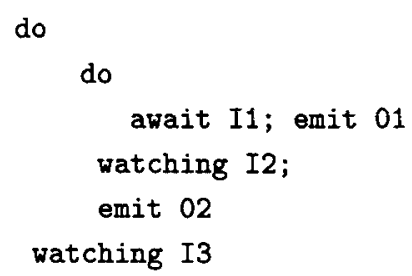

If I1 occurs strictly before I2 and I3, then the internal await statement terminates normally; 01 is emitted, the internal watching terminates since its body terminates, 02 is emitted, and the external watching also terminates since its body does. If I 2 occurs before II or at the same time as it, but strictly before $I 3$, then the internal watching preempts the await statement that should otherwise terminate, 01 is not emitted, 02 is emitted, and the external watching instantaneously terminates. If $I 3$ occurs before $I 1$ and $I 2$ or at the same time as then, then the external watching preempts its body and terminates instantaneously, no signal being emitted. Notice how nesting watching statements provides for priorities.

We can now explain why "await $S$ " is defined as "do halt watching $S$ ". The semantics of halt is simple: it keeps the control forever and never terminates. When $S$ occurs, halt is preempted and the whole construct terminates just as expected. Notice that halt is the only kernel statement that takes time by itself.

The trap-exit construct is similar to an exception handling mechanism, but with purely static scoping and concurrency handling. In "trap $T$ in stat end", the body stat is run normally until it executes an "exit $\mathrm{T}$ " statement. Then execution of stat is preempted and the whole trap construct terminates. The body of a trap statement can contain parallel components; the trap is exited as soon as one of the components executes an "exit T" statement, the other components being preempted. However, exit preemption is weaker than watching preemption, in the sense that concurrent components execute for a last time when exit occurs. Consider for example the statement

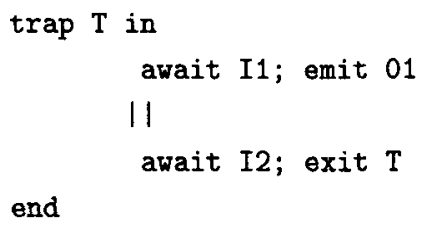

If I1 occurs before I2, then 01 is emitted and one waits for I2 to terminate. If I2 occurs before $I 1$, then the first branch is preempted, the whole statement terminates instantaneously, and 01 will never be emitted. If I 1 and I2 occur simultaneously, then both branches do execute and 01 is emitted. Preemption occurs only after execution at the concerned instant: by exiting a trap, a statement can preempt a concurrent statement, but it does leave it its "last wills". 
Since we accept simultaneity, we must define what it means to exit several traps simultaneously, i.e. define priorities between traps. The rule is simple: only the outermost trap matters, the other ones being discarded. For example, in

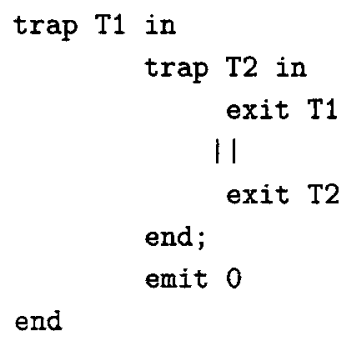

the traps $\mathrm{T} 1$ and $\mathrm{T} 2$ are exited simultaneously, the internal trap $\mathrm{T} 2$ is discarded and 0 is not emitted.

Traps also provide a way of breaking loops, which would otherwise never terminate:

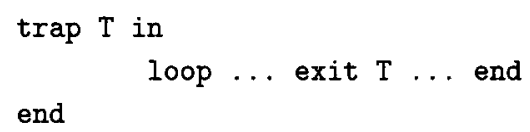

\section{A.3 Value handling}

Since full Esterel will be informally used in the sequel, we briefly describe the way in which values are handled.

Types can be either predefined like integer or be abstract like Time; abstract types are meant to be implemented in the host language in which a program is compiled, $\mathrm{C}$ or ADA for example.

A signal can carry a value of a type declared in the signal declaration. A valued signal has a unique value at each instant. A signal value may change only when the signal is received from the environment or locally emitted with a new value, by executing "emit $S$ (exp) ". The current value of a signal $S$ is accessed at any time by the expression '? S'.

One can declare local variables by the statement

$\operatorname{var} \mathbf{X}$ in stat end

Variables deeply differ from signals by the fact that they cannot be shared by concurrent statements. Variables are updated by instantaneous assignments " $\mathrm{X}:=\exp$ " or by instantaneous side-effecting procedure calls "call $\mathrm{P}(\ldots)$ ", where a procedure $\mathrm{P}$ is an external host-language piece of code that receives both value and reference arguments.

Expressions may involve variables, signal values '? S', and external host-language function calls (external functions must not perform side effects). The computation of an expression is instantaneous. The "if exp then statl else stat 2 end" statement instantaneously tests for the truth of exp.

Finally, occurrence counters can be added to preemption statements, as in "do stat watching $5 \mathrm{~S}$ ". 